\title{
EVALUATION OF SELECTED RESOURCE ALLOCATION AND SCHEDULING METHODS IN HETEROGENEOUS MANY-CORE PROCESSORS AND GRAPHICS PROCESSING UNITS
}

\author{
Milosz CIZNICKI*12, Krzysztof KUROWSKI ${ }^{1}$, Jan WȨGLARZ ${ }^{12}$
}

\begin{abstract}
Heterogeneous many-core computing resources are increasingly popular among users due to their improved performance over homogeneous systems. Many developers have realized that heterogeneous systems, e.g. a combination of a shared memory multi-core CPU machine with massively parallel Graphics Processing Units (GPUs), can provide significant performance opportunities to a wide range of applications. However, the best overall performance can only be achieved if application tasks are efficiently assigned to different types of processor units in time taking into account their specific resource requirements. Additionally, one should note that available heterogeneous resources have been designed as general purpose units, however, with many built-in features accelerating specific application operations. In other words, the same algorithm or application functionality can be implemented as a different task for CPU or GPU. Nevertheless, from the perspective of various evaluation criteria, e.g. the total execution time or energy consumption, we may observe completely different results. Therefore, as tasks can be scheduled and managed in many alternative ways on both many-core CPUs or GPUs and consequently have a huge impact on the overall computing resources performance, there are needs for new and improved resource management techniques. In this paper we discuss results achieved during experimental performance studies of selected task scheduling methods in heterogeneous computing systems. Additionally, we present a new architecture for resource allocation and task scheduling library which provides a generic application programming interface at the operating system level for improving scheduling polices taking into account a diversity of tasks and heterogeneous computing resources characteristics.
\end{abstract}

Keywords: scheduling, resource management, GPUs, many-core computing systems

\footnotetext{
${ }^{1}$ Poznań Supercomputing and Networking Center, Poland

${ }^{2}$ Institute of Computing Science, Poznań University of Technology, Poland

*to whom correspondence should be addressed, e-mail: miloszc@man.poznan.pl
} 


\section{Introduction}

Heterogeneous multi-core and many-core computer systems can provide significant performance opportunities to a wide range of algorithms and legacy applications $[22,18,2,10,11,12]$. However, in order to improve the efficiency of calculations a coordinated use of CPUs and GPUs must be implemented. Many programming languages and parallel execution environments have been proposed till now to help users during processes of designing and managing the execution of applications on heterogeneous computing resources. The most commonly used are Message Passing Interface (MPI) for scalable cluster computing [14] and OpenMP for shared memory multiprocessor systems [9]. In a nutshell, MPI is a programming model where nodes in a computing cluster do not share the memory, all data sharing and tasks interactions must be done through the explicit message passing. MPI has been successfully adapted by many developers over the last two decades in high performance computing reaching the level of hundreds or thousands of simultaneously used computing resources [7]. Various extensions to MPI enabled users also to run large-scale applications in grid environments [16]. The OpenMP is a specification of compiler directives, library routines, and environment variables that provides an easy parallel programming model portable across shared memory computing resources. However, in the advent of GPUs, there is a growing number of developers using a new programming model called CUDA [19]. It has been designed to support joint execution of applications on CPU and GPU computing resources. Aspects of CUDA are similar to both MPI and OpenMPI in such a sense, that a programmer manages the parallel code constructs, although OpenMP does more of the automation in managing parallel execution. There are several ongoing research efforts that aim at automation of parallelism management and performance optimization to the CUDA tool chain $[17,15,8,27]$.

As processor units in a heterogeneous system are often based on entirely different architectures and will continue to evolve with increased numbers of cores and more powerful accelerators, the efficient scheduling and mapping of application tasks to computing resources will be crucial. It is even a bigger challenge to deal with scheduling of a set of tasks or big workloads to underlying heterogeneous computing systems in order to achieve a good overall performance. Therefore, in this paper we compare the efficiency of commonly used tasks management and scheduling approaches as well as propose a set of improvements to better utilize heterogeneous many-core processors and graphics processing units within single nodes. Our experimental tests, benchmarks and analysis were possible due to a new scheduling library we have successfully designed and implemented at the operating system level. The generic purpose scheduling library provided interfaces for different task management and scheduling methods and can be easy adapted by external researchers interested in this topic.

The rest of the paper is organized as follows. In Section 2 the related work dealing with scheduling tasks on heterogeneous computing architectures is presented. Section 3 present main assumptions of the considered scheduling problem. Section 4 explains in more details the scheduling library and its architecture used during benchmarks. 
In Section 5 we present selected task scheduling algorithms used in our comparative studies. Experiment results are presented and discussed in Section 6. Finally, we conclude the paper and present future work in Section 7.

\section{Related work}

Different runtime systems exist today to support management of tasks onto heterogeneous many-core systems consisting of CPUs and GPUs. These systems provide high-level programming interfaces and hide the complexity of underlying management processes, however, many of them require from a programmer to decide how to schedule tasks associated with a given type of processor unit. For instance, the Hybrid API, an extension to CUDA, enables writing parallel Charm ++ applications, and their mapping and execution on heterogeneous CPU/GPU nodes [26]. The Hybrid API provides model for organizing and scheduling work on the GPU. Nevertheless, authors have not released results of their performance benchmarks. An interesting approach is the StarPU runtime system that supports scheduling of tasks on multiple CPUs and GPUs [5]. In a nutshell, StarSS provides a unified execution model for heterogeneous tasks and supports various scheduling algorithms. Additionally, there is an extension to StarSS environment called GPUSs [6]. It provides simple load balancing and data management mechanism for CPUs connected with multiple GPUs. A slightly different approach is promoted by the Anthill runtime system [23]. Anthill is a data flow oriented framework in which applications are decomposed into a set of event driven filters, where for each event the runtime system can use either GPU or CPU for tasks processing. It is worth mentioning RenderAnts which uses work stealing scheduling algorithm to manage tasks on multiple GPUs [28]. Authors improved the performance of the system by duplicating some computations and avoiding timeconsuming data transfers. There is also the Harmony runtime system [13], where applications are composed of code segments invoking computing kernels dispatched on heterogeneous processors.

\section{Scheduling problem}

The problem we consider in the paper is to find the best assignment of application tasks to heterogeneous resources taking into account a given performance criterion. In general, there are two types of scheduling strategies: static and dynamic. In static mapping all tasks are mapped in an off-line planning phase. It means that the mapping is done before the execution. In dynamic mapping all tasks are assigned to resources during the execution. In practice, dynamic mapping is applied when the execution times of tasks can not be accurately estimated at the compilation time. Moreover, dynamic strategies better reflect real-life scenarios where tasks arrive continuously to a heterogeneous system at some arrival rate and all processors in the system do need not to be idle at a given time. As a result, dynamic scheduling strategies need 


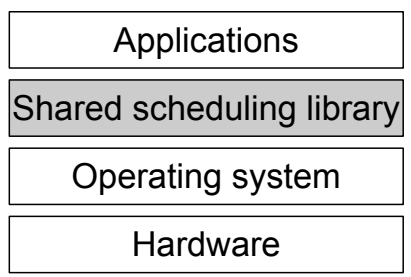

Figure 1: The scheduling system architecture

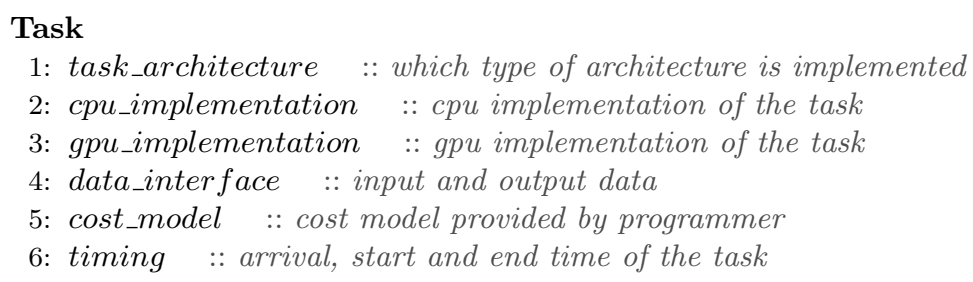

Figure 2: Task structure

to take into account both existing information about all the tasks in the system and the current status of heterogeneous processing units to find a best schedule. Additionally, the dynamic scheduling policies can be divided into two categories: immediate mode and batch mode. In an immediate mode, scheduling policies can only consider a new task for mapping in opposite to a batch mode where a subset of new tasks is considered for mapping. Both advantages and disadvantages of batch and immediate modes are presented in [20]. In a static approach the key scheduling performance metric is makespan. However, this metric is not sufficient for dynamic scheduling where tasks are typically submitted to a heterogeneous system at different rates during different time intervals. Depending on the arrival rates and scheduling strategies a heterogeneous system can go through different saturation during different time intervals. Consequently, it is necessary to analyze the complete behavior of a heterogeneous system under different load conditions and workloads. It is also important to find connection between the dynamic schedules and the performance metrics.

In this paper we consider the dynamic scheduling with immediate mode of tasks on heterogeneous computing resources.

\section{Scheduling system}

In order to perform evaluation studies we have successfully designed and implemented a scheduling system as a dynamic library for the Linux operating system [25]. The library is shared among applications tasks which are competing for the access to heterogeneous resources. Figure 1 gives an idea of the scheduling system architecture. 


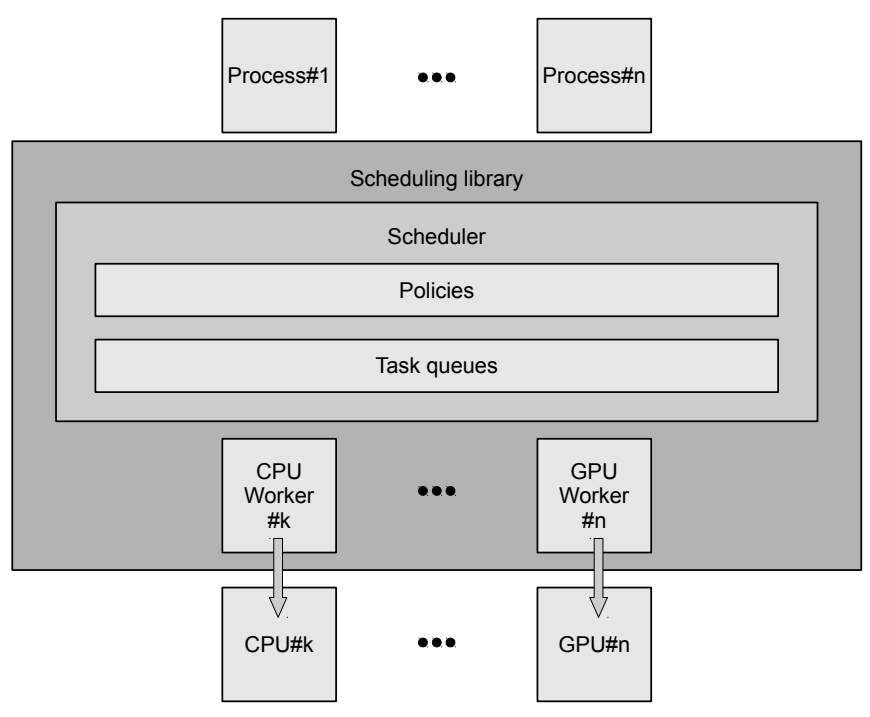

Figure 3: The internal design of the scheduling library

In fact, the scheduling system is an intermediate layer between applications, operating system and hardware (heterogeneous computing resources). In the scheduling system it is assumed that each application task has several implementations, one per each processor type. Figure 2 presents a task structure. The task is defined as a function with all included arguments. It is a basic unit of the scheduling runtime. The tasks input and output arguments have to be provided through data interface. Furthermore, implementations of functions will exhibit different performance depending on a target processor type. Moreover, a performance depends on computation type, since strict sequential task is more convenient for execution on CPU than on GPU. Therefore, the scheduling system exploits additional parameters which are associated with each task. There are two key additional parameters: a cost function and a problem size. The cost function is implemented by the developer and reflexes the complexity of a given task. In fact, for many commonly used libraries there are available performance and cost models and they can help developers to estimate the complexity of their tasks. The another parameter - the problem size gives to the scheduling system more characteristics about task complexity. For instance, a size of the data as the main bottleneck of computing on GPUs is inefficient transferring the data through the PCI Express comparing to other memory buses.

The procedure of dispatching tasks to scheduling library is the following. An application submits task to the scheduler. When the task is ready it is dispatched by the scheduler to the selected processor. After the task is finished the callback is executed.

The scheduling system can be used through the scheduling library by many applications at the same time. Each application can submit many tasks at a time. There is no guaranty on ordering of submitted tasks. One can not assume that task submitted 

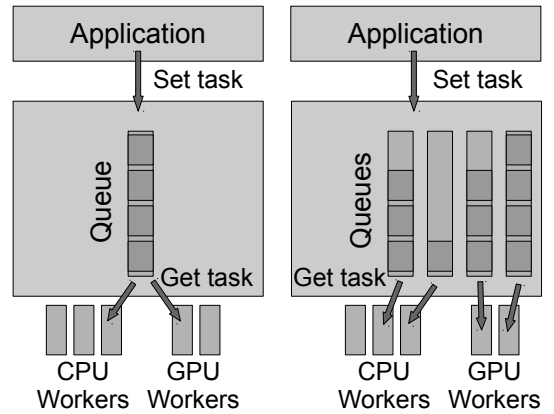

Figure 4: Left - central queue, right - separate queues.

at time $t_{1}$ will be finished before task submitted at time $t_{2}=t_{1}+\Delta t$. However, a programmer can provide a synchronization mechanism through the callback routine. A disadvantage of this method is that the scheduling system can receive penalty in execution time.

Figure 3 shows the internal design of the scheduling library. On the top there are applications which submit tasks. Tasks are scheduled by selected scheduling policy on a set of queues. Afterwards, workers pop tasks from queues. A worker is a thread associated with a specific processor. In conclusion, the worker owns an abstract queue of tasks. Two operations are possible on each queue: a task submission and request for a task to execute. As mentioned before, tasks are submitted by scheduler and requested by workers. Queues are shared among workers and a mechanism protecting queue from concurrent access is implemented.

A generic scheduler can not achieve the best performance for all kinds of tasks. Consequently, our scheduling system supports several scheduling policies, as presented in Section 5. The scheduling system provides a uniform interface to design the scheduling policies. Furthermore, it is implemented in high abstract level regardless of the underlying target architecture. The scheduling policies can be developed independently from applications. Consequently, one can easily add a new scheduling policy. Moreover, it is possible to change actual scheduling policy at runtime. The role of a scheduling policy is to dispatch task to the selected processor. The application has to submit tasks to the library. Afterwards, tasks are prepared for execution. Subsequently, tasks are dispatched to the task queue. Finally, tasks can be balanced between queues and distributed to the processors. It depends on the scheduling policy, if there is one queue for all processors or separate queue for each processor, as shown in Figure 4. The scheduling policy is responsible for creating a set of queues and assorting them with workers. The queue can be implemented by various data structures such as FIFO or Stack. In a scheduler one can distinguish different topologies of queues. For instance, one queue type is a main queue which is shared among workers. Another type is a per worker queue which holds tasks intended for a particular worker. Further type is a priority queue where tasks with equal priority are scheduled to the same queue.

In this work the scheduling policies implement a greedy list scheduling paradigm. It arises from the flow of the tasks in the scheduling system. The tasks are in general 


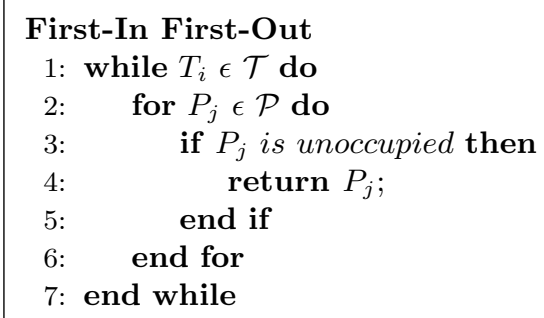

Figure 5: FCFS policy

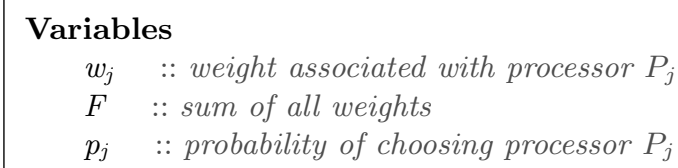

\section{Random selection with weights}

1: for $P_{j} \in \mathcal{P}$ do

2: $\quad P_{j} \leftarrow w_{j}$

3: end for

4: $F=\sum_{i=1}^{n} w_{j}$

5: for $P_{j} \in \mathcal{P}$ do

6: $\quad p_{j}=w_{j} / F$

7: $\quad P_{j} \leftarrow p_{j}$

8: end for

9: while $T_{i} \in \mathcal{T}$ do

10: randomize $\mathbf{r} \epsilon[0,1)$

11: $\quad$ choose $P_{j}$, where $\sum_{i=1}^{i=j-1} p_{i} \leq \mathbf{r}<\sum_{i=1}^{i=j} p_{i}$

12: end while

Figure 6: Random selection with weights policy

inserted on one of the queues, later on the scheduling decisions are not reconsidered. As a consequence, the system is restricted to the list scheduling and is less general. However, it provides simplicity and portability of implementing new scheduling policies. Moreover, it is transparent to the applications and workers.

\section{$5 \quad$ Resource allocation and scheduling algorithms}

\subsection{First Come First Served}

First Come First Served (FCFS) policy is based on greedy concept. There is one task queue $Q_{\text {central }}$ which is shared among all workers. As a result accesses to the queue have to be synchronized. The task $T_{i}$ which comes to the system is distributed to 
the first unoccupied processor $P_{j}$. If there are more than one unoccupied processor than it is unspecified which processor will receive the task. On the other hand, if all workers are occupied the task waits for the first free worker. The algorithm of the FCFS policy is shown in Figure 5.

\subsection{Random Selection with Weights}

In this policy each worker is associated with a separate task queue. What is more, each worker posses weight $w_{j}$ which defines the speed of the processor $P_{j}$. When new task arrives to the system, the processor $P_{j}$ is selected with probability proportional to its weight $w_{j}$. Therefore, processor with a higher weight has more chance to be selected. The weights associated with processors can be set by a programmer before running the scheduling system. The algorithm of Random Selection with Weights is presented on Figure 6. In lines 1-3 each processor receives defined weight. Next, the sum $F$ of weights is calculated (line 4 ) and probability $p_{j}$ of choosing the processor $P_{j}$ based on the weight $w_{j}$ is calculated (lines 5-8). The random number $r \epsilon[0,1)$ with an uniform distribution is generated (line 10) for the incoming task $T_{i}$. Finally, based on this number the processor $P_{j}$ which receives the task is chosen (line 11).

\section{$5.3 \quad$ Work-Stealing}

Following resource allocation policy is Work-Stealing [4]. Similarly to Random Selection with Weights each worker is associated with a separate task queue. The tasks are assigned to the processors in a Round-Robin manner. The policy selects the processor $P_{j}$ pointed by a counter. After assigning the task $T_{i}$ to the processor $P_{j}$ the policy increments the counter. If the end of the processor list is reached it starts from the beginning. If the processor $P_{j}$ finishes all its tasks it tries to lock other queue $Q_{k}$, where $k \neq j$, and steal task $T_{i}$. The algorithm of work-stealing policy is presented in Figure 7. In lines 2-4 the tasks are distributed among the queues with the RoundRobin resource allocation policy. If some processor finish all its tasks from it tries to lock other queue and steal the task (lines 5-13).

\subsection{Heterogeneous-Earliest-Finish-Time}

The last scheduling policy is the Heterogeneous Earliest Finish Time algorithm described in [24]. Before presenting details of the scheduling policy it is necessary to define the EST and EFT attributes, which are derived from a given partial schedule. $\operatorname{EST}\left(T_{i}, P_{j}\right)$ and $\operatorname{EFT}\left(T_{i}, P_{j}\right)$ are the Earliest Execution Start Time and the Earliest Execution Finish Time of the task $T_{i}$ on the processor $P_{j}$ respectively. For the first task $T_{1}$ :

$$
\operatorname{EST}\left(T_{1}, P_{j}\right)=0
$$




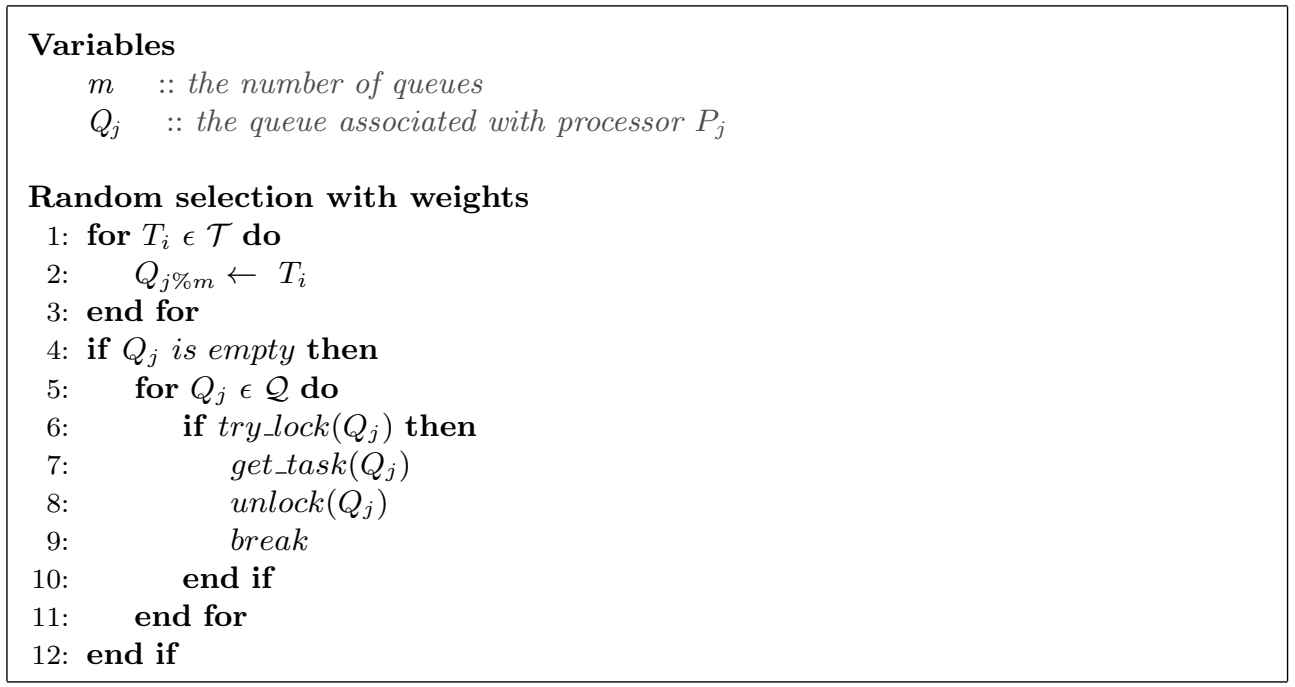

Figure 7: Work-stealing policy

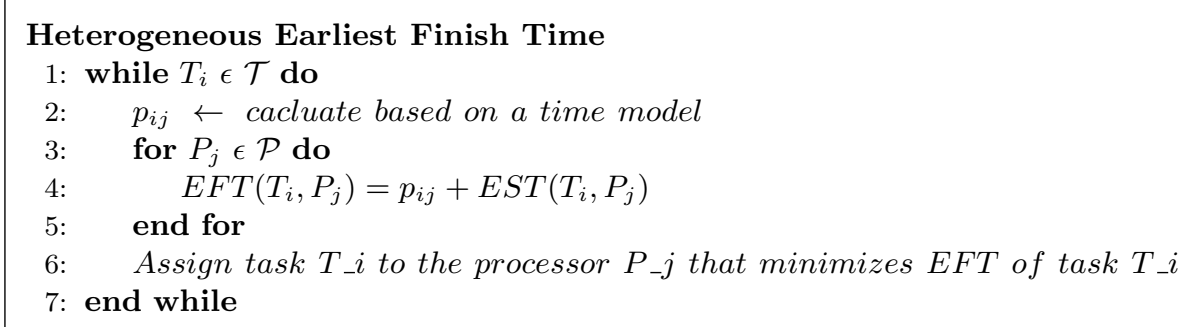

Figure 8: HEFT policy

$$
\begin{gathered}
\operatorname{EST}\left(T_{i}, P_{j}\right)=\operatorname{avail}[j] \\
\operatorname{EFT}\left(T_{i}, P_{j}\right)=p_{i, j}+\operatorname{EST}\left(T_{i}, P_{j}\right)
\end{gathered}
$$

For the next tasks the EFT and EST values are computed recursively. Starting from the first task as shown in Equations 1,2 and 3. The value avail $[j]$ is the earliest time at which the processor $P_{j}$ is ready to execute the task. If $T_{i}$ is the last assigned task on processor $P_{j}$ then avail $[j]$ is the time that processor $P_{j}$ completed the execution of the task $T_{i}$ and it is ready to execute next task.

The HEFT algorithm schedules each arrived task on the processor which minimizes the task's finish time. The algorithm is presented in Figure 8. When task arrives to the system the vector of processing times $\left[p_{1 j}, p_{2 j}, \ldots, p_{m j}\right]^{T}$ is calculated based on the size $s_{i}$ of the task (line 2). The developer can provide an execution time model for each processor type to estimate the execution time of the task. After the execution time is calculated, the $\operatorname{EFT}\left(T_{i}, P_{k}\right)$ value between task $T_{i}$ and all processors $P_{j} \epsilon \mathcal{P}$ is 


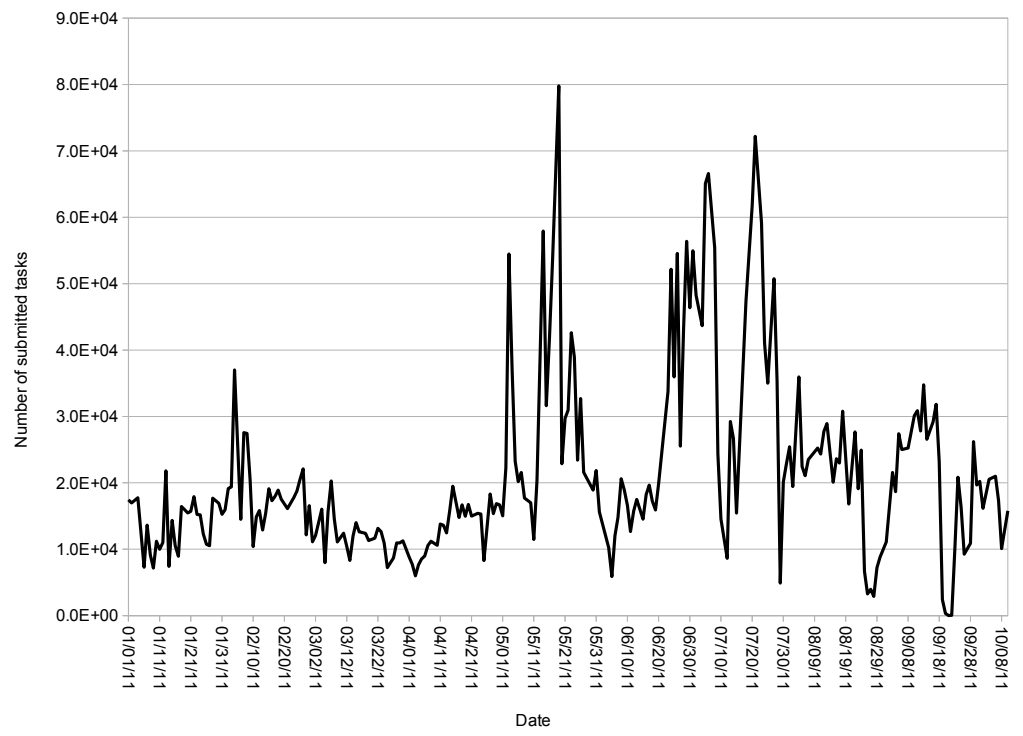

Figure 9: Workload of the Zeus cluster.

calculated (line 3-5). Finally the task $T_{i}$ is assigned to the processor $P_{j}$ that minimizes the EFT of task $T_{i}$ (line 6 ).

\section{Results}

\subsection{Simulation setup}

The parameters used in our experimental studies are based on the real workload from the heterogeneous computing cluster to reflect a real scenario [1]. All jobs were submitted to the cluster are scheduled by the Torque resource management system [21]. The whole resource management system has been divided into queues that have associated set of constraints. These constraints can limit the size and length of each job or available processor types. Workload data from the Torque resource management system are used to obtain simulation parameters. These data include nine months of work (from 2011.01.01 to 2011.10.01). Figure 9 shows the number of submitted tasks on all queues each day. The mean number of submitted tasks is 17243.

For the experimental studies we assumed that the heterogeneous computing node is equipped with six CPU cores and two GPUs. The 1000 tasks are simulated for a period of 16 hours. For each scheduling policy 50 simulations have been executed. Within 
the simulation period the arrival times $r_{i}$ of the tasks are randomly generated using the Poisson distribution. To simulate an overloaded system the mean arrival time of the task is equal to 5 seconds. The execution times of all tasks are generated using the gamma distribution method described in [3] to simulate the task and processor heterogeneity. The four different cases of the task and processor heterogeneity are considered during experiments: the high task and high processor heterogeneity (hh), the high task and low processor heterogeneity (hl), the low task and high processor heterogeneity (lh) and the low task and low processor heterogeneity (ll).

$$
V=\sigma / \mu
$$

Let $\sigma$ and $\mu$ be the standard deviation and mean respectively of a set of the execution times. The coefficient of variation $V$ is defined as in Equation 4 . The coefficient of variation expresses the standard deviation as a percentage of the mean of the values. All task and processor heterogeneity cases use a mean execution time $\mu$ of the task equal to 480 seconds. For the high task and high processor heterogeneity the $V$ is equal to 0.9 , whereas for the low task and low processor heterogeneity the $V$ is equal to 0.3. In our case the two types of processors are used: CPUs and GPUs. The simulated execution time $p_{i j}$ of the task $T_{i}$ on the processor $P_{j}$ is connected with its size $s_{i}$. The simulated execution times are not known to the scheduler, however the sizes $s_{i}$ are known. The size $s_{i}$ of the task is computed using $p_{i j}$ as the mean execution time and a $V$ equal to 0.1 .

Figure 10 shows how a gamma density function changes with the $V$ parameter. When the $V$ parameter increases from 0.3 to 0.9 the shape of the distribution changes from a bell-shaped curve to the curve biased to the left. The dispersion of the execution times is more spread and a domain is more than twice as large. The value of the $V$ parameter represents the heterogeneity of the tasks and processors.

\subsection{Experimental results}

The obtained results during our experiments are shown in Figures 11 and 12 for the following criteria:

- schedule length $C_{\max }=\max \left\{C_{i}\right\}$,

- mean flow time $\bar{F}=\frac{1}{n} \sum_{j=1}^{n} F_{i}$,

where $C_{i}$ is a completion time and $F_{i}=C_{i}-r_{i}$ is a flow time of task $T_{i}$.

Each figure consists of four scenarios for all combinations of high and low task and processor heterogeneity.

For all the heterogeneity cases the Heterogeneous Earliest Finish Time (HEFT) policy achieved the shortest schedule lengths, while the Random Selection with Weights (RWW) policy produced very long schedules. The RWW policy associated higher weight $w_{i}$ with GPU processor type and in consequence tasks were mainly submitted to GPU processor types. As the execution times of the tasks are gamma distributed, 


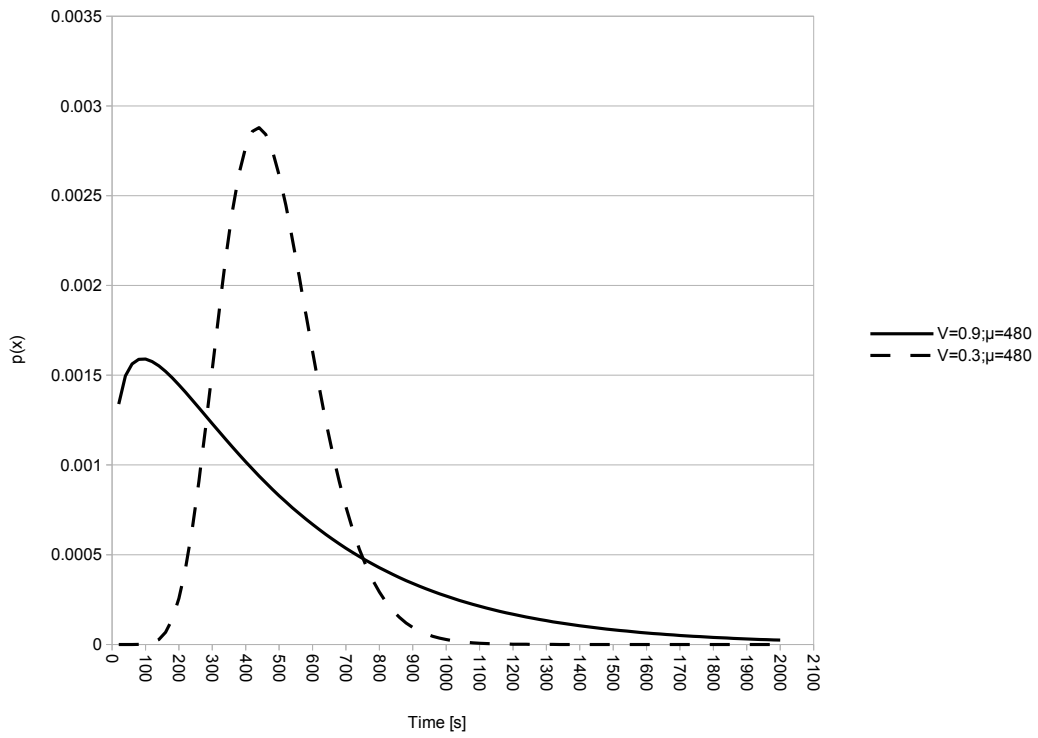

Figure 10: Gamma probability density function for $(V=0.3, \mu=480)$ and $(V=$ $0.9, \mu=480)$

there is no significant improvement in the schedule length. What is even more important, there is performance degradation comparing to the basic FCFS policy. One can use the RWW policy to model the execution times of the tasks bound to the specific processor type. For instance, if submitted tasks better scale on GPUs, it can be modeled by assigning to GPU higher weight $w_{j}$. For low heterogeneity cases, FCFS, WS and HEFT scheduling policies obtained similar results.

However, for high heterogeneity cases we observed significant differences in the behavior of above-mentioned scheduling policies. It turned out that the HEFT policy can better accommodate the differences in task execution times for different processor types. The HEFT policy tries to estimate the execution time of the task based on its size $s_{i}$ and submit it to an appropriate processor $P_{i}$. Additionally, there is no significant difference in schedule length for both high and low heterogeneity cases between the FCFS and WS policy. Each scheduling policy provides similar schedule lengths. Both scheduling policies are based on greedy model, each worker associated with processor requests task from queue. However, when the execution times of the tasks are very short the schedule length between the FCFS and WS policy may be significantly different. One queue in the FCFS policy may force processors to become idle while waiting for access to the queue to fetch the next task. 


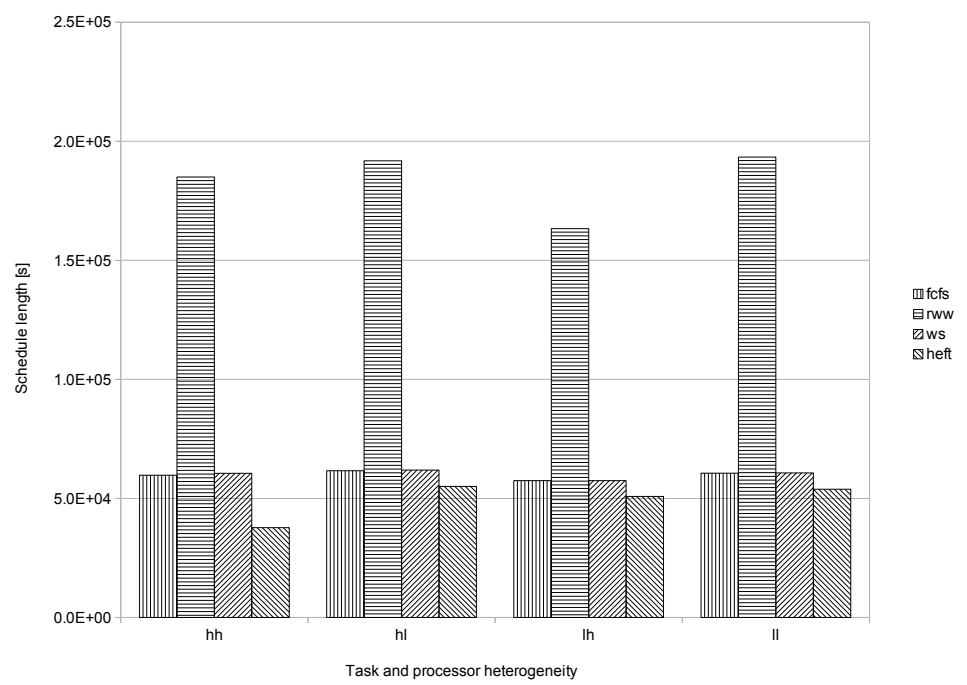

Figure 11: The simulation results for schedule length $C_{\max }$ : hh) high task and high processor heterogeneity, hl) high task and low processor heterogeneity, lh) low task and high processor heterogeneity, ll) low task and low processor heterogeneity

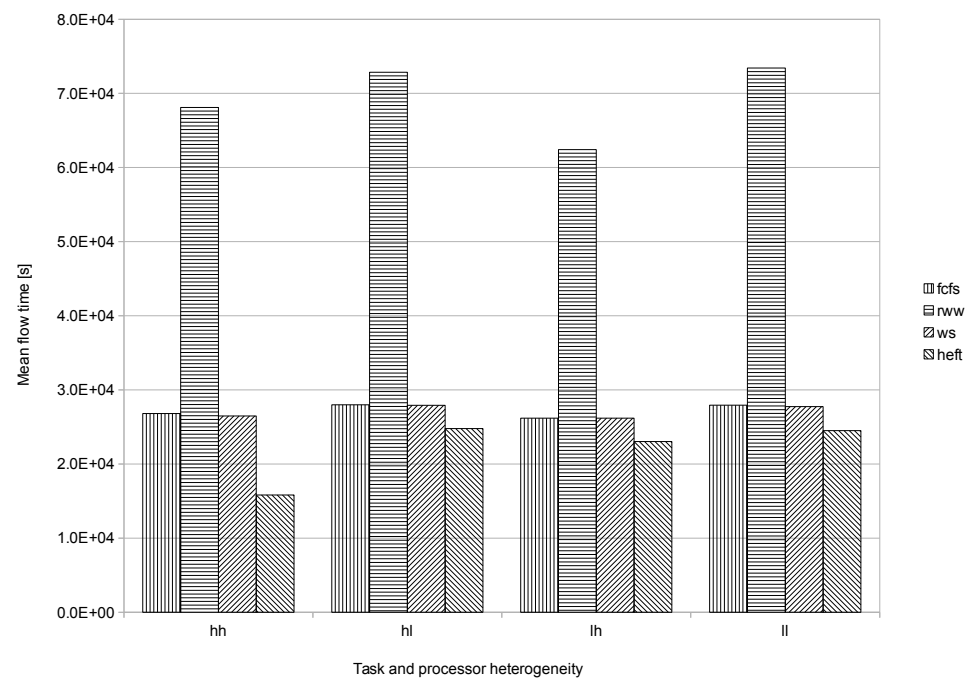

Figure 12: The simulation results for mean flow time $\bar{F}$ : hh) high task and high processor heterogeneity, hl) high task and low processor heterogeneity, lh) low task and high processor heterogeneity, ll) low task and low processor heterogeneity 


\section{Conclusions and future work}

The presented scheduling system was designed and developed for the heterogeneous computing resources that consist of two types of processors CPUs and GPUs. It transparently handled various task distributions over heterogeneous processors. Additionally, we have evaluated the overall performance of four scheduling policies during experimental studies. In our studies we used the synthetic workload constructed on real data obtained from log files in the heterogeneous computing cluster. Obtained results indicate that carefully selected scheduling policies to the level of heterogeneity of tasks and resources can improve significantly the overall performance. In our setup the HEFT policy gave the best results up to $60 \%$ in schedule length comparing to the simple FCFS policy. Further improvements will be also possible if the HEFT policy is able to take into account additional task parameters and characteristics.

In our future work we are planing to extend the scheduling system with poweraware scheduling policies. We assume that new power-aware programming and execution models together with domain specific application tools must be designed and verified to support a higher-level abstraction of parallelism independently from rapidly changing underlying computing architectures. Those new models should assist the code development process, including parallel code generation, coupling, faulttolerance, scheduling and management of running applications up to the exascale level. Moreover, new models and algorithms should be adaptive and dynamically configurable to meet domain-specific application requirements as well as help developers to fully exploit heterogeneous hardware features.

\section{Acknowledgments}

This work has been supported by the National Science Centre (NCN) in Poland under the MAESTRO grant No DEC-2013/08/A/ST6/00296.

\section{References}

[1] (2014). Specification of the zeus cluster, http://www.top500.org/system/177388.

[2] Agullo, E., Demmel, J., Dongarra, J., Hadri, B., Kurzak, J., and Langou, J. (2009). Numerical linear algebra on emerging architectures: The PLASMA and MAGMA projects. Journal of Physics: Conference Series, 180:12-37.

[3] Ali, S., Siegel, H., Maheswaran, M., Hensgen, D., and Ali, S. (2000). Representing task and machine heterogeneities for heterogeneous computing systems. Tamkang Journal of Science and Engineering, 3(3):195-208.

[4] Arora, N., Blumofe, R., and Plaxton, C. (1998). Thread scheduling for multiprogrammed multiprocessors. In Proceedings of the tenth annual ACM symposium on Parallel algorithms and architectures, pages 119-129. ACM. 
[5] Augonnet, C., Thibault, S., and Namyst, R. (2010). StarPU: a Runtime System for Scheduling Tasks over Accelerator-Based Multicore Machines.

[6] Ayguadé, E., Badia, R., Igual, F., Labarta, J., Mayo, R., and Quintana-Ortí, E. (2009). An extension of the StarSs programming model for platforms with multiple GPUs. Euro-Par 2009 Parallel Processing, pages 851-862.

[7] Blazewicz, M., Brandt, S., Kierzynka, M., Kurowski, K., Ludwiczak, B., Tao, J., and Weglarz, J. (2011). CaKernel - A parallel application programming framework for heterogenous computing architectures. Scientific Programming, 4:185-197.

[8] Blazewicz, M., Hinder, I., Koppelman, D., Brandt, S., Ciznicki, M., Kierzynka, M., Loffler, F., Schnetter, E., and Tao, J. (2013). From physics model to results: An optimizing framework for cross-architecture code generation. Scientific Programming, 21(1):1-16.

[9] Chapman, B., Jost, G., and Van Der Pas, R. (2008). Using OpenMP: portable shared memory parallel programming, volume 10. MIT press.

[10] Ciznicki, M., Kierzynka, M., Kopta, P., Kurowski, K., and Gepner, P. (2012a). Benchmarking data and compute intensive applications on modern CPU and GPU architectures. In Procedia Computer Science 9, volume 9, pages 1900-1909.

[11] Ciznicki, M., Kierzynka, M., Kurowski, K., Ludwiczak, B., Napierala, K., and Placzynski, J. (2012b). Efficient isosurface extraction using marching tetrahedra and histogram pyramids on multiple GPUs. In Parallel Processing and Applied Mathematics, pages 343-352. Springer Berlin Heidelberg.

[12] Ciznicki, M., Kopta, P., Kulczewski, M., Kurowski, K., and Gepner, P. (2014). Elliptic solver performance evaluation on modern hardware architectures. In Parallel Processing and Applied Mathematics, pages 155-165. Springer Berlin Heidelberg.

[13] Diamos, G. and Yalamanchili, S. (2008). Harmony: an execution model and runtime for heterogeneous many core systems. In Proceedings of the 17th international symposium on High performance distributed computing, pages 197-200. ACM.

[14] Gropp, W., Lusk, W., and Skjellum, A. (1999). Using MPI: portable parallel programming with the message-passing interface, volume 1 . MIT press.

[15] Kamil, S., Chan, C., Oliker, L., Shalf, J., and Williams, S. (2010). An autotuning framework for parallel multicore stencil computations. Parallel $\&$ Distributed Processing, pages $1-12$.

[16] Kurowski, K., Oleksiak, A., and Weglarz, J. (2013). Multicriteria, multi-user scheduling in grids with advance reservation. Journal of Scheduling, 13 (5):493508 .

[17] Lee, S., Min, S. J., and Eigenmann, R. (2009). OpenMP to GPGPU: a compiler framework for automatic translation and optimization. ACM Sigplan Notices, 44.4:101-110. 
[18] Linderman, M., Collins, J., Wang, H., and Meng, T. (2008). Merge: a programming model for heterogeneous multi-core systems. ACM SIGOPS operating systems review, 42 .

[19] Nickolls, J., Buck, I., Garland, M., and Skadron, K. (2008). Scalable parallel programming with cuda. Queue, 2:40-53.

[20] Shoukat, M., Maheswaran, M., Ali, S., Siegel, H., Hensgen, D., and Freund, R. (1999). Dynamic mapping of a class of independent tasks onto heterogeneous computing systems. In Journal of Parallel and Distributed Computing. Citeseer.

[21] Staples, G. (2006). Torque resource manager. In Proceedings of the 2006 ACM/IEEE Conference on Supercomputing, SC '06, New York, NY, USA. ACM.

[22] Stone, J., Gohara, D., and Shi, G. (2010). OpenCL: A parallel programming standard for heterogeneous computing systems. Computing in science \& engineering, 12.3:66.

[23] Teodoro, G., Sachetto, R., Sertel, O., Gurcan, M., Meira, W., Catalyurek, U., and Ferreira, R., editors (2009). Coordinating the use of GPU and CPU for improving performance of compute intensive applications. IEEE.

[24] Topcuoglu, H., Hariri, S., and Wu, M. (2002). Performance-effective and lowcomplexity task scheduling for heterogeneous computing. IEEE transactions on parallel and distributed systems, pages 260-274.

[25] Torvalds, L. (1999). The linux edge. Communications of the ACM, 42(4):38-39.

[26] Wesolowski, L. (2008). An application programming interface for general purpose graphics processing units in an asynchronous runtime system. Master's thesis, Dept. of Computer Science, University of Illionois.

[27] Wienke, S., Springer, P., Terboven, C., and an Mey, D. (2012). OpenACC - first experiences with real-world applications. Euro-Par 2012 Parallel Processing, pages 859-870.

[28] Zhou, K., Hou, Q., Ren, Z., Gong, M., Sun, X., and Guo, B. (2009). RenderAnts: interactive Reyes rendering on GPUs. In ACM Transactions on Graphics (TOG), volume 28 , page 155 . ACM.

Received July, 2014 